\title{
Optimizing the Performance Evaluation of Robotic Arms with the Aid of Genetic Algorithm
}

\author{
K Shivaprakash Reddy \\ Assistant Professor, Department of Mechanical \\ Engineering, The Oxford College of Engineering, \\ Bommanahalli, Hosur Road, \\ Bangalore - 560068, Karnataka, India
}

\author{
B. Durgaprasad \\ Phd, Professor, Department of Mechanical \\ Engineering, \\ JNTU College of Engineering, Jawaharlal Nehru \\ Technological University, Anantapur - 515002, \\ Andhrapradesh, India
}

\author{
PVK Perumal \\ Phd, Professor and Head \\ Department of Aeronautical Engineering, \\ M.A.M School of Engineering, Trichy - Chennai \\ Trunk Road, Siruganur, Trichy -621105 , \\ Tamil Nadu, India \\ M.A Murtaza \\ Phd, Professor, Department of Mechanical \\ Engineering, \\ The Oxford College of Engineering, Bommanahalli, \\ Hosur Road, Bangalore - 560068, \\ Karnataka, India
}

\begin{abstract}
In recent decades, the robotic system is becoming more and more significant and this leads the humans to perform precarious tasks at secure distances. In this paper, we planned to improve the performance of robotic gear box. Like our human hand, the robot hands are utilized for performing various tasks. To facilitate the task, flexible robotic arms is necessary to improve the performance of robotic arm gear box. In existing research, only static parameters were considered to make the robotic arms as flexible but such parameter itself is not enough to obtain the optimized value for the gear box to perform. In order to attain the optimized value for the robotic arm gear box, we considered both dynamic and static parameters. By using such parameter values, the optimized value for the gear box is obtained, which result in better gear box performance. Thus, our proposed work fills the gap occurs in the existing works and this paves the way for obtaining flexible robotic arms. Our result obtained by considering various parameters has shown better performance than the existing works.
\end{abstract}

\section{Keyword}

Robotic arm, flexible, Static \& Dynamic parameters, Gearbox, Genetic algorithm.

\section{INTRODUCTION}

Robots are corporal agents that achieve tasks by manipulating the physical world. Normally, robots are equipped with sensors to sense the environment and effectors to claim physical forces on it. Industrial robots play a major role in grinding method in the automation process. Most of the grinding robots work in a constrained environment, where simultaneous position and force control is vital [1]. Robots can be classified into three main categories: (1) Manipulators, (2) Mobile robots, and (3) Humanoid robots [2]. Robots find intensive applications in factories to enhance product quality and safety, while minimizing costs and processing time. The robot model depends on the inertia, mass, and center of mass of each link [3]. Robotics is based on two prominent technologies: Telemanipulators and the capacity of numerical control of machines. Tele-manipulators are remotely controlled machines that often hold an arm and a gripper. The movements of arm and gripper will take place according to the instructions given by the humans through his/her control device. Numeric control allows controlling of machines very accurately with respect to a given coordinate system [2].

\section{1. Types of Robot}

\section{Mobile Robots}

The mobile robots use a special group of effectors for locomotion, such as wheels, tracks, and legs. The differential drive contains two independently actuated wheels, one on each side. The robot travels in a straight line when the movement of both wheels is at same velocity. If they move in opposite directions, then the robot turns on the spot. The development of mobile robots was motivated by the desire to automate transportation in production processes and autonomous transport systems. New types of mobile robots have been created recently like insectoid robots with several legs modeled after examples nature gave us or independent robots for underwater usage.

\section{Hard Working Robots}

Usually, robots have been used to replace human workers in areas of difficult toil, which is structured enough for mechanization, like assembly line work in the automobile industry (the classical example) or harvesting machines in the agricultural zone. A few existing examples apart from the assembly robot are Melon harvester robot, Ore transport robot for mines, robot that removes paint from big ships and a robot that creates high precision sewer maps. If robot is used in a proper environment, then it can work faster, cheaper and more exact than human beings.

\section{Transporters}

Most autonomous transport robots still desire ecological changes to find their way, although they are already widely in use. But, designing a robot that can navigate using natural landmarks is probably an end to science fiction. Examples of currently available transporters are (1) Container transporters employed to load and unload cargo ships, (2) Medicine and 
food transport systems in hospices, and (3) Autonomous helicopters employed to transport goods to distant areas.

\section{Insensible Steel Giants}

Robots are used in perilous, toxic or nuclear environments because they can be easily protected against hazardous environments and are adequately replaceable. In some places, robots have been used for cleaning up a mess. For example, in Chernobyl disaster, robots have helped to clean up the nuclear waste, and also robots are employed to clean grenades and mines all around the world. Moreover, robots are sent to Mars and into the depth of the oceans. They can also explore sunken ships and can walk on the craters of active volcanoes.

\section{Servants and Toys}

Robots may not yet be a common sight in our world, but we already meet and used them in several places. A lot of modern toys like the Sony Aibo are spoiling the today's children's life. Robots are mainly invented to help the aged people to have a better and more secure life. Today, they begin to come with us in the name of toys or household helpers [2].

\section{Industrial Robots}

For more than thirty years, industrial robots have been entrenched in the manufacturing area, used for performing the tasks such as stacking, casting, painting, sorting, welding, component soldering and more. This use context highlights the core value proposition of an industrial robot: performing tasks incessantly and precisely in work environments and scales difficult for humans. Industrial robots, developed for performing operations swiftly, continually and precisely have a long heritage in the manufacturing industry, working in relatively static environments and in large numbers. The oil and gas industry suggest the use of industrial robotics to enhance the safety and efficiency as well as to decrease the environmental impact. New developments in regions that are tricky or perilous for humans to work in could be easily handled and maintained by remotely-controlled industrial robots [4].

\section{2. Gear-Box}

A gearbox is used to change the speed from the low rotating rotor to the high rotating generator [5]. Robotic systems that need to provide high torques at the end effectors usually contain high reduction gears in their transmissions, causing some gearspecific friction components to appear, such as position dependent friction. Since this force happens once a pair of teeth comes together, it produces a periodic waveform friction with the frequency by which the two teeth match. Thus, it has been always considered as position dependent friction.

The following parameters are considered for examining the function of gears,
a) Direction of turning
b) Relative speed
c) The number of revolutions
d) Mechanical advantages concerning the principles of power and speed [6]

When the power is transmitted along the line of action direction, friction takes place along the off line-of-action direction, which lies orthogonal to the line of action. The main reason for this friction is that the teeth slide together instead of rolling perfectly. Hence, meshing friction is a source of incompetence in gearboxes. Along the line of action, the power and load in gear trains are transmitted. The relative reduced rigidity of shaft support ball bearings may be responsible for small shaft displacements along the line-of-action direction, which would lead to torque oscillations and this is called position dependent friction. However, the meshing friction force in gear teeth is transmitted in the off line-of-action direction. The friction coefficient between the gear teeth greatly depends on lubricant properties, and it decreases when the relative sliding velocity between gear teeth increases.

\section{3. Types of Gears}

The amplitude of the oscillation caused by meshing friction also depends heavily on the gear type. The working principle of spur or helical gears is rolling between teeth and hence, the meshing friction could be small in some cases. But, the working principle of other gear types, such as worm gears, is almost pure sliding friction. When speed is low, the lubricant film is inadequate to prevent contact between asperities, and friction becomes very high. Only if speed increases, the lubricant film will become adequate to reduce friction [7].

Some of the gear types are,

External \& internal gears

$>$ Spur gear

Helical gears

$>$ Skew gears

$>$ Double helical gears

$>$ Bevel gears

$>$ Hypoid gears

Crown gears

$>$ Worm gear

$>$ Non-circular gears

$>$ Rack and pinion gearing

$>$ Epicyclic gearing

Sun and planet gearing

$>$ Harmonic drive gearing

Cage gear

\section{4. Common Gearbox Parameters}

Some of the common gear-box parameters are,

\section{Viscous Friction Coefficient}

Viscous friction between two surfaces that have relative motion between them relies on dimensional parameters such as contact area and clearance between the two surfaces, and also relies on fluid properties, such as fluid specific gravity and viscosity. Viscous friction is inversely proportional to the clearance between the two meeting surfaces [8].

\section{Coulomb Friction Coefficient}

Coulomb friction is a basic measurement of the friction force that exists between two dry surfaces in contact with each other. The coulomb friction coefficient is a static force, which is somewhat higher than motive force when two materials are at rest while in contact with each other. This coefficient of friction is eminent for several simple, pure materials and is given as a unit-less number. For dry surfaces, the coefficient of friction for 
wood against concrete is 0.62 , for polystyrene against steel is 0.3 to 0.35 , and for steel against Teflon is 0.04 . These numbers are utilized to compute the force required to conquer static friction, called as the friction force, by multiplying the coefficient of friction times the normal force. The normal force is the mass of the materials times' gravitational pull, with vector calculations added in if the two surfaces are moving up or down an incline against gravitation pull, or towards it [9]

\section{Striebeck Friction Coefficient}

Striebeck methodically studies the variation of friction between two liquid lubricated surfaces as a function of a dimensionless lubrication parameter $\eta \mathrm{N} / \mathrm{P}$, where $\eta$ denotes the dynamic viscosity, $\mathrm{N}$ represents the speed i.e., revolutions per minute of a bearing, and $\mathrm{P}$ represents the load projected on to the geometrical surface [10].

\section{Friction Smoothness Coefficient}

Friction is the resistance that an object encounters when moving over another (OED). It is quick and easy to drag an object over glass than sandpaper because the sandpaper has more frictional resistance. In many situations, it was assumed that a surface does not use any frictional force if it is "smooth". In real life, however, this wouldn't be the case. A "rough" surface is one that provides some frictional resistance [11].

\section{Total Moment of Inertia}

In traditional mechanics, moment of inertia, also called mass moment of inertia, rotational inertia, polar moment of inertia of mass, or the angular mass, (SI units $\mathrm{kg} \cdot \mathrm{m}^{2}$ ) is a measure of an object's resistance to any change in its state of rotation. It is the inertia of a rotating body corresponding to its rotation. The moment of inertia plays much the same role in rotational dynamics as mass does in linear dynamics, depicting the relationship between angular momentum and angular velocity, torque and angular acceleration, and numerous other quantities. The symbol 'I' and sometimes ' $\mathrm{J}$ ' are often used to represent the moment of inertia or polar moment of inertia [12].

In this paper, our foremost intent is to reduce the conflict occurs between the theoretical value and the practically obtained experimented value by considering the parameter values. By utilizing genetic algorithm, we obtain the optimized parameter value, which in case certainly reduce the error occurred and also the result obtained after applying the optimized parameter value almost bring the intended value and experimentally obtained value.

\section{RELATED WORKS}

Heterogeneity is one of the most noticeable features of robotic applications. Large robotic projects engage numerous different researchers with a variety of hardware and software that must be integrated efficiently to develop applications that not only satisfy classic robotic requirements but also software engineering aspects. However, most prior solutions to this problem either do not cope with such heterogeneity or do not embrace specific robotic requirements. In 2008, Juan-Antonio Fernandez-Madrigal et al. [13] have proposed a framework for the implementation of heterogeneous robotic software via a software engineering technique: the BABEL development system, intended to cover the main phases of the application lifecycle such as, design, implementation, testing, and maintainance when unavoidable heterogeneity conditions were present. The potency of proposed system was revealed by its support for designing and implementing different real robotic applications that employs various programming languages $(\mathrm{C}$,
C++, JAVA), execution platforms (RT-operating systems, MSWindows, no operating system at all), communication middleware (CORBA, TCP/IP, USB), and also various hardware components (PC, microcontrollers, and a wide variety of sensor and actuator devices in mobile robots and manipulator arms).

In 2008, Sungho Jo [14] has proposed a biologically inspired robotic model, which combines modified feedback error learning, an unsupervised learning, and the viscoelastic actuator system in order to drive adaptive arm motions, and also discussed the potential utility of a biomimetic design of robot skill. The feedback error learning was reliable with the cerebellar adaptation, the unsupervised learning, the synergy network adaptation, and the viscoelastic system of the muscles. The redundant actuators were controlled efficiently by applying a feed forward adaptive approach in the low dimensional control space and an adaptive synergy distribution. The amalgamation of the two adaptive control approaches was evaluated by controlling a two-link planar robot arm with six muscular actuators in the gravitational field. The simulationbased study has revealed that the control method can adjust the robot arm motions swiftly and robustly to make smooth, human-like motions.

Flexible robot manipulators have abundant advantages over their rigid counterparts. They have increased payload-to weight ratio, they operate at higher speeds, employ less energy and smaller actuators, and they are secure during interaction with their environments. Conversely, light design along with external effects result in components which can oscillate with extreme amplitudes. These oscillations cause deviation from the desired path and long idle periods between tasks in order to perform the intended operation securely and precisely. In 2008, Abdullah Ozer et al. [15] have examined the capability of a vibration control method for a two-link flexible robotic arm. The excessive oscillations were controlled by using variable stiffness control (VSC) method. Due to its dissipative nature, the method was stable and it was relatively insensitive to significant parameter changes and suitable to be implemented on existing robots. Their research considers that the source of the flexibility was either the joints or the links or both. Simulation results were presented to exhibit the flexibility of the proposed control method. Experiments were conducted on a laboratory prototype and the results were presented to prove the validity of simulations.

According to the task involved, a human performs a variety of adroit movements by adjusting the dynamic characteristics of his/her musculoskeletal system. Mechanical impedance parameters can be used to represent such characteristics of human movements. If the regulation mechanism of human impedance properties during the task can be clarified and modeled, there is a chance that human skillful strategies can be included into robot motion control. In 2008, Toshio Tsuji et al. [16] have studied the human hand impedance in preparation for task operations, the so-called "task-readiness impedance", in a virtual ball-catching task. They have also discussed a biomimetic impedance control of robotic manipulators for contact tasks by computer simulations using measured task-readiness impedance.

In 2009, Freeman et al. [17] have provided an experimental test facility for use by stroke patients in order to enhance the sensory-motor function of their upper limb. Subjects were seated at the workstation and their task is to continually follow reaching trajectories that are projected onto a target above their arm. To perform this, they used a voluntary control with the addition of electrical stimulation mediated by advanced control 
methods applied to muscles in their impaired shoulder and arm. The particulars regarding the design of workstation and its periphery systems were given, together with a depiction of its use during the healing of stroke patients.

In 2009, Yunquan Sun et al. [18] have performed robotic belt grinding operations by placing a work piece to the end effector and commanding it to travel along a path while maintaining contact with the belt grinding wheel. A constant contact force throughout the grinding process was essential to give a smooth finish on the work piece, but it was tricky to maintain this force due to a multitude of installation, manipulation, and calibration errors. The proposed method for robotic belt grinding was described, which mainly concentrates on system calibration and force control to enhance grinding performance. The overall theory was explained and the experimental results of turbine blade grinding for each step of the proposed method were shown.

Antagonistic Driven Compliant Joints (ADCJs) are object, drawn substantial attention in current robotics research, representing one of the most extensively applied solutions to develop human-like and safe joints for human-robot communication. In 2010, Nicola Vitiello et al. [19] have introduced a sensor less torque control technique, appropriate for ADCJs actuated robots. The proposed technique follows two steps: (i) off-line characterization of the flexibility of the actuation units, defined by the force-elongation curve, and (ii) online estimation of the force exerted by each actuation unit, through a direct measure of the joint angle, and of the "resting position" of each actuation unit. The proposed force estimation technique was employed to create two autonomous force controllers, which can be then fused to control the resulting joint torque, with no need of additional torque sensors. The performance of the proposed torque control was analyzed over the shoulder and the elbow ADCJs of the 2-link 2-DOFs planar robotic arm NEURARM. The technique was proved to work effectively, achieving better performances on the test platform, and represents a suitable alternative to modern sensor-based torque controls.

In 2011, Celso De La Cruz et al. [20] have proposed an energetic model of a robotic wheel chair considering a lateral deviation of the center of mass. The Lyapunov and input/output stability theories were utilized to create a tracking and positioning adaptive control for the robotic wheel chair. Properties of the dynamic model regarding to its matrices and parameters were exhibited. A filter was employed to obtain a closed loop equation that permits designing of adaptive control law. Subsequently, a projection algorithm was used to enhance the adaptive control in the sense of eliminating the parameter drift. Experimental results have shown the enhanced performance of the adaptive control.

In 2011, C.M. Wronka et al. [21] have proposed a dynamic model of a robotic manipulator mounted on a moving base by means of the Euler-Lagrange technique. It was assumed that the base inertia was large enough not to be affected by the manipulator motion and hence can be treated as a time-varying parameter in the dynamic equations. The presented derivation was applied to a Mitsubishi PA10-6CE robotic manipulator mounted on a 2 -DOF platform. The model was evaluated by comparing simple closed-loop control results of the simulated model with experimental data from the manipulator mounted on the platform.

Achieving manipulation tasks interactively in real environments necessitates a high level of precision and stability. At the same time, when one cannot assume a fully deterministic and static environment, one must provide the robot with the skill to react quickly to abrupt changes in the environment. In 2012, Ashwini Shukla et al. [22] have recorded the kinematics of arm and fingers of human subjects during unperturbed and perturbed reach and grasp motions. In the perturbed demonstrations, the target's location was altered abruptly after the onset of the motion. Data has shown a strong combination between the hand transport and finger motions. They theorize that the coupling allows the subject to seamlessly and rapidly adapt the finger motion in coordination with the hand posture. To provide their robot with high aptitude, a coupled dynamical system based controller was proposed, whereby two dynamical systems driving the hand and finger motions were coupled. The proposed method has provided a compact encoding for reachto-grasp motions that assures fast adaptation with zero latency for re-planning. From the simulation performed on the real iCub robot, they have proved that the coupling ensures smooth and "human-like" motions.

\section{PROPOSED METHOD}

In this rapid emergent world, robotics plays a vital role in Education, Science and Engineering, medical, and so on. Robotics is mandatory for the secure hand of human to accomplish their progression. In this paper, we concentrate on analyzing the robotic arm gear box to improve their performance by considering various parameters. We utilize Genetic Algorithm (GA) for the performance improvement of robotic arm gearbox. GA is utilized to predict the optimized value among the different parameters. The optimized value is then used to predict the output angular velocity of the motor.

\subsection{Genetic Algorithm Based Optimized Parameters Value}

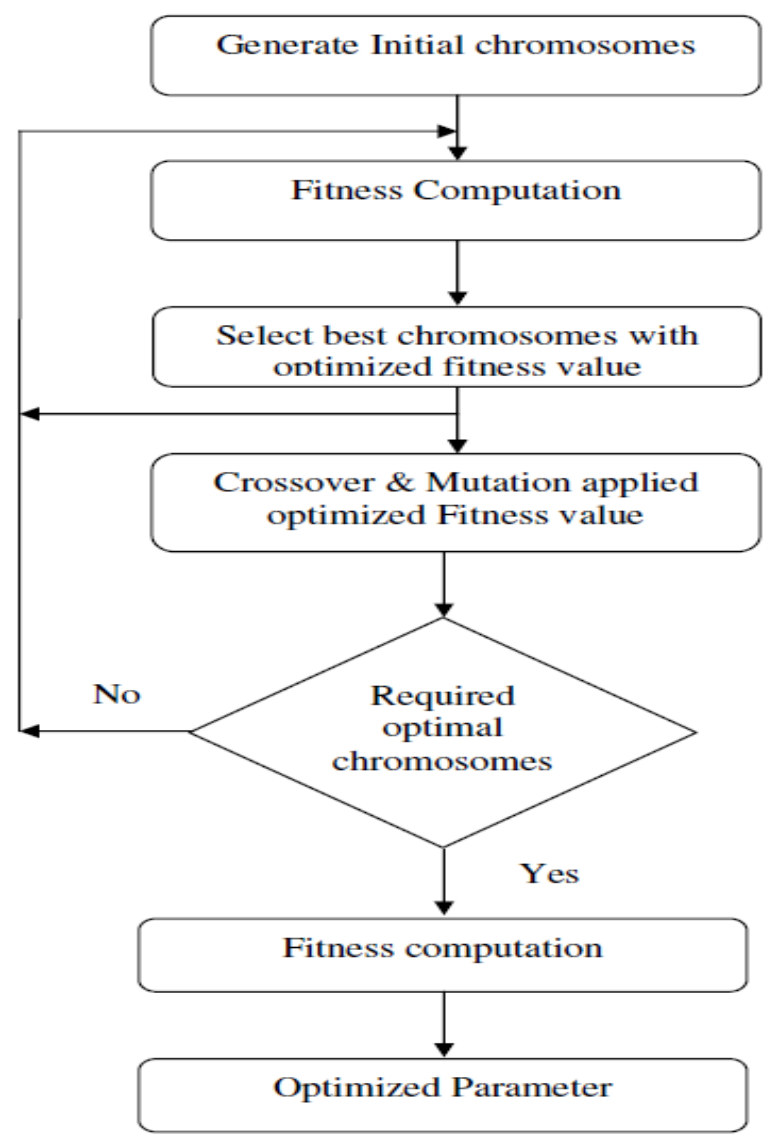




\subsubsection{Generation of Chromosomes}

Initially, $N_{i}$ numbers of chromosomes are generated randomly. Each chromosome has $N_{j}$ number of genes. The randomly generated chromosomes can be determined as,

$$
C_{j}^{(k)}=\left\{c_{0}^{(k)}, c_{1}^{(k)}, c_{2}^{(k)}, \Lambda, c_{N-1}^{(k)}\right\}
$$

Here, $N_{i}$ - No of Chromosomes, $N_{j}$ - No of genes in Chromosomes, and every $j^{\text {th }}$ value has its own range, which should be different from the range of other genes, and the minimized range will give better result when compared to the existing work.

\section{Fitness Computation}

To compute the fitness, all the obtained chromosomes and the corresponding parameter values are taken from the eqn (1). Here, error minimization is the fitness function, which is shown below.

\begin{tabular}{|c|}
\hline $\begin{array}{l}\operatorname{Ere}_{\boldsymbol{e}}=(\mathbf{E x}-\mathrm{Ov}) \\
s=\sum_{m=1}^{N_{m}} E r_{e}^{m} \\
\psi=\frac{s}{N_{m}} \\
\quad \text { If } \min (\psi) \\
\quad \text { Select the chromosome } \\
\text { End If }\end{array}$ \\
\hline
\end{tabular}

Where, Ex - Experimental value; Ov - model value ; $E r_{e}$ Error Element ; s- sum of error Elements ; $N_{e}$ - No of Elements; $\psi$ - Mean of error elements, which is also said to be Fitness. From the above pseudo code, select $N_{X}$ number of chromosomes to be applied with the genetic operations such as, crossover and mutation.

\section{Objective Function}

$$
\text { \& }(t)=\left[\begin{array}{c}
x_{3}-x_{4} \\
x_{4}-x_{5} \\
\frac{1}{I_{m}}\left(-T_{s t}\left(x_{1}\right)-D_{g}\left(x_{3}-x_{4}\right)-T_{f r}\left(x_{3}\right)+u\right) \\
\left.T_{s t}\left(x_{1}\right)+D_{g}\left(x_{3}-x_{4}\right)-k_{s} x_{2}-D_{a}\left(x_{4}-x_{5}\right)\right) \\
\frac{1}{I_{a}}\left(k_{s} x_{2}+D_{a}\left(x_{4}-x_{5}\right)\right) \\
I=I_{a}+I_{m}+I_{g}
\end{array}\right]
$$

$I_{\boldsymbol{m}}=$ Moment of inertia of motor

$I_{\boldsymbol{a}}=$ Moment of inertia of arm

$I g=$ Moment of inertia of gear box

$\boldsymbol{T}_{\boldsymbol{S t}}=$ Spring Torque

$D_{a}=$ Damping Parameters of arm

$D_{g}=$ Damping parameters of gearbox

$k_{S}=$ Stiffness of the second spring

$T_{f r}\left(x_{3}\right)-C_{v} x_{3}+\left(C_{c}+C_{c s} \operatorname{sech}\left(\sigma x_{3}\right)\right) \tanh \left(t x_{3}\right)$

$$
\begin{aligned}
& C_{v}=\text { Viscous friction coefficient } \\
& C_{C}=\text { Coulomb friction coefficient } \\
& C_{C S}=\text { Striebeck friction coefficient. } \\
& T_{f r}=\text { Friction torque } \\
& \quad T_{s t}\left(x_{1}\right)=k_{g b_{1}} x_{1}+k_{g b_{3} x^{3} 1} \\
& k g b_{1}=\text { Gearbox stiffness parameter } 1 \\
& k_{g b_{2}}=\text { Gearbox stiffness parameter } 2 \\
& T_{S t}=\text { Spring Torque } \\
& \qquad E(\theta)=\frac{1}{N} \sum_{t=1}^{N} e^{2}(t, \theta)
\end{aligned}
$$

$\mathrm{E}$ is said to be the Error value

$$
e(t, \theta)=y(t)-y(\hat{t}, \theta)
$$

If $\hat{y}=(t / \theta)$, then the simulated output of the model is obtained with the input $\mathrm{u}(\mathrm{t})$ and without $\mathrm{e}(\mathrm{t})$ for the current parameter vector $\theta$. The criterion (6) is minimized by an iterative numerical search algorithm, which involves simulation of the system for different values of $\theta$.

$$
\min _{\theta} E(\theta)=\frac{1}{N} \sum_{t=1}^{N} e^{2}(t, \theta)
$$

\section{Crossover and Mutation}

Crossover and mutation are the two significant genetic operators of the genetic algorithm. Among the dissimilar sort of crossover, the two-point crossover is affianced here at a crossover rate $\mathrm{Cr}_{t}$. In the two-point crossover, two points are chosen on the parent chromosomes by means of the equations (3) and (4). The genes in between the two points $C_{S 1}$ and $C_{s 2}$ are then interchanged between the parent chromosomes to obtain $N_{p / 2}$ children chromosomes. The crossover points $C_{s 1}$ and $C_{s 2}$ are determined as,

$$
\begin{gathered}
C s_{1}=\frac{\left|C_{j}^{(k)}\right|}{N_{j}} \\
C s_{2}=C s_{1}+\frac{\left|C_{j}^{(k)}\right|}{N_{j}}
\end{gathered}
$$

The child chromosomes are obtained and then the mutation operation is performed on these chromosomes. This process is repetitive until the minimized error is obtained concerning to threshold value, which is supposed to be the best chromosome and sort those sets in rising order and then evaluates them by means of its error value.

\section{Selection of Best Chromosomes}

After the completion of $I t r_{\max }$ number of iterations, the best chromosome is chosen from the obtained chromosomes. Here, the best chromosome is one having least error value. Subsequently, the genes of best chromosome are arranged in the rising order and the chromosome that has least error is preferred as the best gene.

From the above process, we have obtained the optimal fitness value i.e., eqn (8). By utilizing this fitness value, we can obtain the value similar to that of the experimental value. 


\section{RESULTS AND DISCUSSION}

To obtain the optimized fitness value, Genetic Algorithm is used which is more preferable for optimization process. Here, we use optimize fitness value for four set of experiments (i.e.,) Estimation, Validation1, Validation2, Validation 3, which gives more accurate value than the standard value. The table 1 shows that the proposed fitness value is greater than that of the existing fitness value and the corresponding fitness comparison graph is shown in the following figure. 1. The standard and existing data are obtained from [23]. The figure 2, 3, 4, and 5 illustrates the general graph model for proposed and existing work that represent how much percentage of blue color has occurred in the black color shaded graph. The remaining portion shows that the existing work is lag with the standard value.

Table 1. Comparison of Existing fitness value and proposed fitness value

\begin{tabular}{|c|c|c|}
\hline Experiments & $\begin{array}{c}\text { Existing Fitness } \\
\text { Value }\end{array}$ & $\begin{array}{c}\text { Proposed } \\
\text { Fitness Value }\end{array}$ \\
\hline Estimation & 72.63 & 78.7 \\
\hline Validation 1 & 73.22 & 79.2 \\
\hline Validation 2 & 8.887 & 37.5 \\
\hline Validation 3 & 95.86 & 98 \\
\hline
\end{tabular}

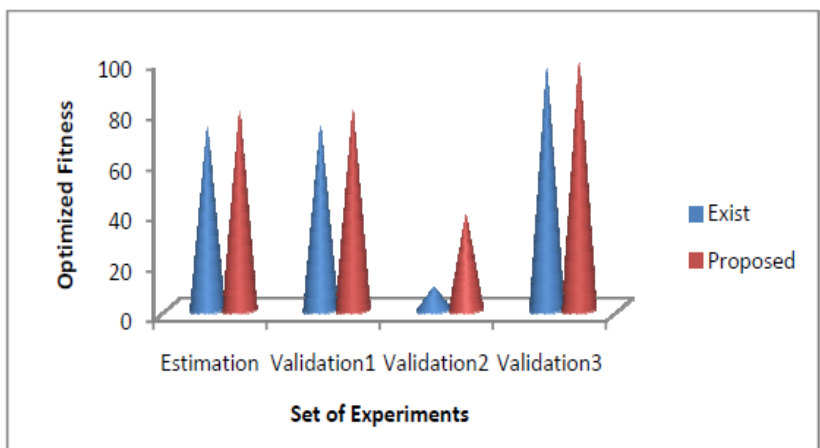

Figure.1 (Fitness comparison)

The above graph clearly explains that our proposed technique has achieved greater value than existing work. This is proved from four set of experiments i.e., Estimation, Validation 1, Validation 2, and Validation 3. The aforementioned graph is plotted from the obtained fitness value for existing work and proposed work.

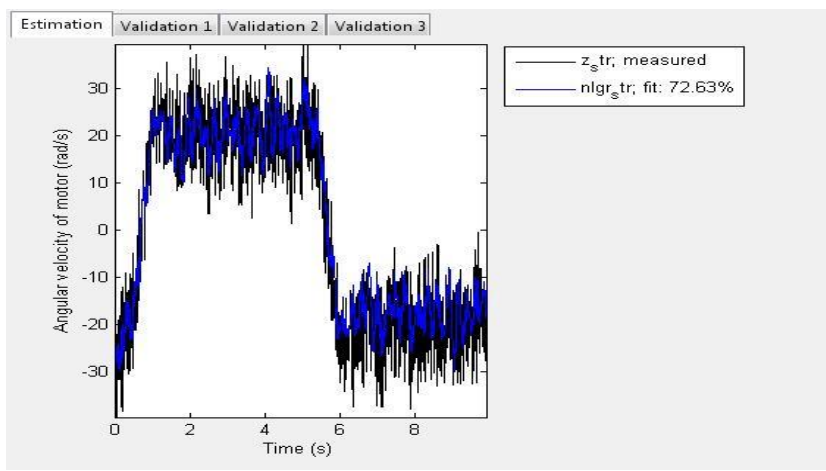

Figure.2 (Estimation)

During estimation, $72.63 \%$ of fitness value is obtained for existing work (i.e.,) the blue color has occurred only $72.63 \%$ in the black shaded part in the graph. But in our proposed work, we attained $78.7 \%$ fitness value, which is higher than that of existing work.

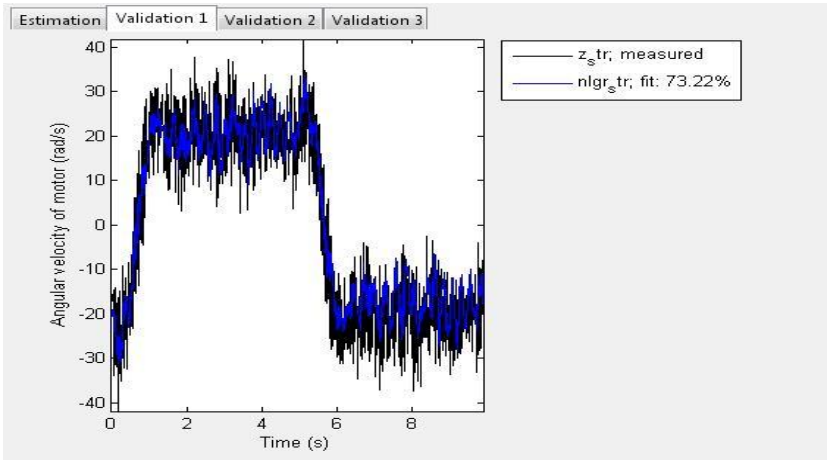

Figure.3 (Validation 1)

For validation $1,73.22 \%$ of fitness value is obtained for existing work when compared to that of standard value (i.e.) the blue color has occurred only $73.22 \%$ in the black shaded part in the graph. But in our proposed work, we attained $79.2 \%$ fitness value which is higher than that of existing work.

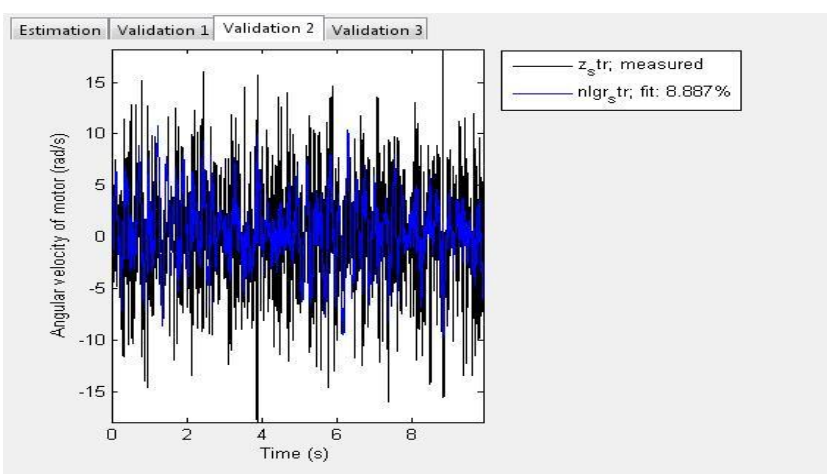

Figure.4 (Validation 2)

For validation $2,8.887 \%$ of fitness value is obtained for existing work (i.e.) the blue color has occurred only $8.887 \%$ in the black shaded part in the graph. But in our proposed work, we attained $37.5 \%$ fitness value which is higher than that of existing work.

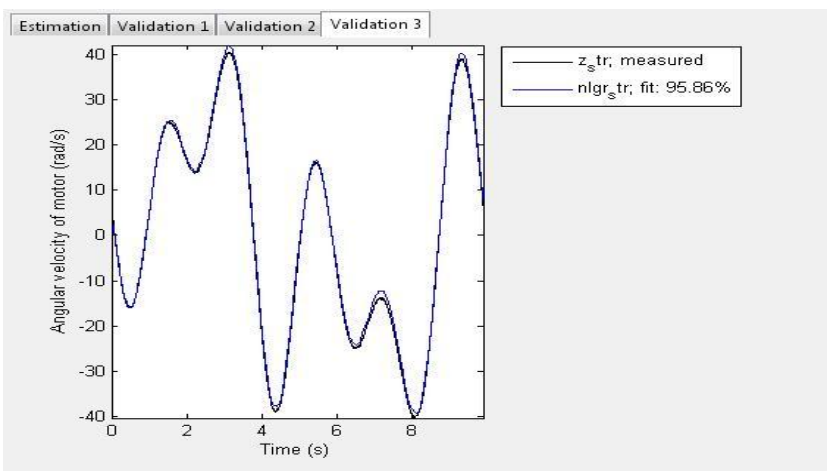

Figure.5 (Validation 3)

For validation $3,95.86 \%$ of fitness value is obtained for existing work (i.e.) the blue color has occurred only $95.86 \%$ in the black shaded part in the graph. In our proposed work, we attained 98\% fitness value which is higher than that of existing work. 


\section{CONCLUSION}

A robust technique was proposed to predict the best parameter value from the generated chromosomes. This process was carried out using the data from an experimental industrial robot with a flexible model. The estimated physical parameters by this model have realistic numerical values. But in practices there should be some conflict between practically obtained value and theoretically predicted value. Here, we found that this optimized parameter value of proposed method was similar to that of the existing experimental parameter value and the theoretically obtained value. This incase certainly reduce the error value. This kind of optimized parameter value will definitely improve the flexibility of robotic arms.

\section{REFERENCES}

[1] Saeid Nahavandi, Mohammad Jashim Uddin, Yasuo Nasu, Hieu Trinh., and Mozafar Saadat, "Automated Robotic Grinding By Low-Powered Manipulator" ,Robotics and Computer-Integrated Manufacturing, Vol.23, pp.589598,2007 .

[2] Tim Niemueller., and Sumedha Widyadharma," Artificial Intelligence - An Introduction to Robotics", AI-Robotics, July 8, 2003.

[3] Basilio Bona., and Aldo Curatella," Identification of Industrial Robot Parameters for Advanced Model-Based Controllers Design", Proceedings of the 2005 IEEE International Conference on, Robotics and Automation (ICRA), pp 1681 - 1686, 2005.

[4] Clint Heyer,"Human-Robot Interaction and Future Industrial Robotics Applications", IEEE/RSJ International Conference on Intelligent Robots and Systems (IROS), 2010.

[5] Svend Gade, Richard Schlombs, Christoph Hundeck., and Christian Fenselau," Operational Modal Analysis on a Wind Turbine Gearbox", Conference \& Exposition on Structural Dynamics, 2009.

[6] Joan M. Chambers, Mike Carbonaro., and Hana Murray, "Developing Conceptual Understanding of Mechanical Advantage Through the Use of Lego Robotic Technology", Australasian Journal of Educational Technology, v24 n4 p387-401 2008.

[7] S.E. Hodges., and R.J.Richards,"Look and Learn: Towards Cheap, Flexible Robots", Proceedings of the International Conference on Recent Advances in Mechatronics ICRAM 1995.

[8] Medhat K., and Bahr Khalil," Estimated Versus Calculated Viscous Friction Coefficient in Spool Valve Modeling", Technical Conference on IFPE, 2008.

[9] Coulomb Friction (http://www.wisegeek.com/what-iscoulomb-friction.html)

[10] Tribology (http://en.wikipedia.org/wiki/Tribology).
[11] The Coefficient of Friction (http://www.mathsrevision.net/alevel/pages.php?page=79)

[12] Moment of inertia (http://en.wikipedia.org/wiki/Moment_of_inertia).

[13] Juan-Antonio Ferna' ndez-Madrigal, Cipriano Galindo, Javier Gonza' lez, Elena Cruz-Martin., and Ana CruzMarti'n, "A Software Engineering Approach for the Development of Heterogeneous Robotic Applications" ,Robotics and Computer-Integrated Manufacturing,Vol.24,pp.150-166,2008.

[14] Sungho Jo, "Adaptive Biomimetic Control of Robot Arm Motions" ,Neurocomputing, Vol.71, pp.3625-3630, 2008.

[15] Abdullah Ozer., and S. Eren Semercigil, "An Event-Based Vibration Control for a Two-Link Flexible Robotic Arm: Numerical and Experimental Observations", Journal of Sound and Vibration, Vol.313, pp.375-394, 2008.

[16] Toshio Tsuji., and Yoshiyuki Tanaka, "Bio-Mimetic Impedance Control of Robotic Manipulator for Dynamic Contact Tasks", Robotics and Autonomous Systems, Vol.56, pp.306-316, 2008

[17] C.T. Freeman, Hughes, Burridge, Chappell, Lewin., and Rogers, "A Robotic Workstation for Stroke Rehabilitation of the Upper Extremity Using Fes", Medical Engineering \& Physics, Vol.31, pp.364-373, 2009.

[18] Yunquan Sun, DavidJ. Giblin., and Kazem Kazerounian, "Accurate Robotic Belt Grinding of Work Pieces With Complex Geometries Using Relative Calibration Techniques", Robotics and Computer-Integrated Manufacturing, Vol.25, pp.204-210, 2009.

[19] Nicola Vitiello, Tommaso Lenzi, Stefano Marco Maria De Rossi, Stefano Roccella., and Maria Chiara Carrozza, "A Sensor Less Torque Control for Antagonistic Driven ,Compliant Joints" ,Mechatronics,Vol.20,pp.355-36,2010.

[20] Celso De LaCruz, Teodiano Freire Bastos., and Ricardo Carelli, "Adaptive Motion Control Law of a Robotic Wheel Chair" Control Engineering Practice,Vol.19,pp.113-125,2011.

[21] C.M. Wronka., and M.W. Dunnigan, "Derivation and Analysis of a Dynamic Model of a Robotic Manipulator on a Moving Base", Robotics and Autonomous Systems, Vol.59, pp.758-769, 2011.

[22] Ashwini Shukla., and Aude Billard, "Coupled Dynamical System Based Arm-Hand Grasping Model for Learning Fast Adaptation Strategies", Robotics and Autonomous Systems, Vol.60, pp.424-440, 2012

[23] Erik Wernholt., and Svante Gunnarsson,"Nonlinear Identification of a Physically Parameterized Robot Model", LiTH-ISY-R-2739, pp.1-6, Aug 2006. 